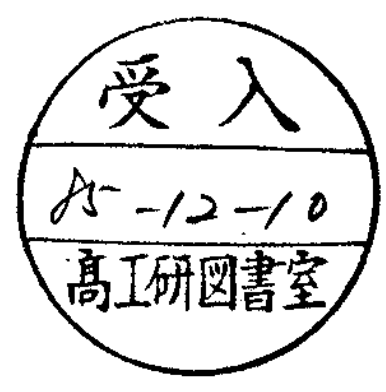

CERN - TH. $-4272 / 85$

\title{
BEAUTIFUL MASS PREDICTIONS FROM SCALAR LATTICE QCD
}

\author{
by \\ Stuart Samuel ${ }^{1}$ \\ CERN-Geneva \\ and \\ Kevin J. M. Moriarty \\ Department of Mathematics, Statistics, and Computing Science \\ Dalhousie University \\ Halifax, Canada B3H 4H8
}

\begin{abstract}
Scalar lattice QCD methods are used to accurately predict the masses of hadrons with beauty, that is, states which contain a b quark. These states have not yet been seen in the laboratory. The accuracy of the predictions $(\approx 25 \mathrm{MeV})$ make the experimentalists.
\end{abstract}

${ }^{2}$ A Sloan Foundation Fellow; On leave of absence from City College of New York, Physics Depart-
ment, 138th and Convent Ave NY, USA 10031

CERN - TH. $-4272 / 85$

November, 1985 
The purpose of this letter is to present the first mass predictions originating from lattice gauge theories. Presently, the only hadron with beauty which has been detected is the $\mathrm{B}(5272)$. Over the next few years it should be possible to produce other beautiful states. Using lattice scalar QCD, we will present predictions accurate to $15-35 \mathrm{MeV}$ for their masses.

Lattice Quantum ChromoDynamics (QCD) presently constitutes the best hope of solving the strong interaction problem. Invented by Wilson over ten years ago [1], it first gave credibility to the idea that QCD confines. Via strong coupling expansions, Wilson manifestly observed quark confinement. The lattice is very good at revealing the strong coupling and long distance properties of the theory, but also important is whether lattice QCD possesses the correct weak coupling behavior, because to take the continuum limit and reconstruct continuum QCD requires seeing lattice asymptotic freedom. It was Creutz [2] who first saw this after Monte Carlo methods were introduced Creutz, Jacobs and Rebbi [3].

These qualitative successes have spurred efforts to obtain quantitative results. Attempts include hadron masses [4-18], magnetic moments $[8,19,20]$, decay constants $[11,12]$, weak interaction processes $[21,22]$ and hadron coupling constants [23]. One successful quantitative result is an accurate measurement of $f_{\pi}$ $[13,14,17,24,25]$. For the most part, however, results have been somewhat disappointing. Hadron mass calculations disagree with experiment in the $10-20 \%$ range (sometimes even more). Little splitting is seen between the nucleon and $\Delta$ (typically $50 \mathrm{MeV}$ versus the experimental amount of $293 \mathrm{MeV}$ ) and the nucleon to $\rho$ ratio is too large. The potential quark models and bag models are still numerically more successful, although they entail ad hoc assumptions and sometimes involve many adjustable parameters.

One should realize, however, that there are certain approximations and difficulties which enter in lattice QCD calculations. There is the quenched approximation $[4,5]$, in which quark loops and "sea" quarks are neglected. Large lattices are needed to avoid finite size effects which creates huge demands on computer resources (time, memory and money). Thus, many calculations have had finite size effects. Other projects have avoided these by working very far away from the physical quark masses. Results are obtained for several quark mass values and extrapolated to physical values. These extrapolations can introduce sizeable errors. Finally, there is the difficulty with lattice fermions [26-28]. The naive prescription of putting fermions on a lattice does not work. Other procedures have to be adopted which make lattice fermions less like their continuum counterparts. Although the lattice is good for the long distance physics, the lattice cutoff can spoil the short-distance physics. This is particularly unfortunate for the spin - spin interaction responsible for splitting such states as the $\pi$ and $\rho$ and the nucleon and $\Delta$. Being short - ranged, it is likely to get distorted and may be the reason for the lack of splitting seen between the nucleon and $\Delta$. 
Recently, some steps [29] has been taken to avoid these difficulties. In order to work at the physical $u, d$, and s quark masses, large lattices were used. It was also advantageous to work further into strong coupling than is conventionally done. This was made possible because an action different from Wilson's was used, the twoparameter action [30] with negative adjoint coupling:

$$
A=\sum_{\square}\left(\left(\beta_{f} / 6\right) \operatorname{tr}\left(U_{\square}+h . c .\right)+\left(\beta_{a} / 9\right)\left|\operatorname{tr}\left(U_{\square}\right)\right|^{2}\right)
$$

where, in our computation, $\beta_{\mathrm{f}}=7.52$ and $\beta_{\mathrm{a}}=-3.321$. In the two-parameter plane, the Wilson theory sits close to a phase transition which manifests itself as a huge bump in the specific heat. It is responsible [31] for the scaling violations $[32-36]$ seen in the cross - over region. By going below the Wilson axis, one is farther from this phase transition, the bump in the specific heat is smaller [37], and there is improvement in the scaling behavior. Excellent scaling has been seen in the string tension [38], the static quark potential [38], wavefunctions $[39,40]$ and hadron masses $[41,42]$. This permitted, for the first time, computations to be carried out at physical quark mass values.

Next, in order to avoid the distortion of the spin - spin interaction, spin degrees of freedom were treated perturbatively. There is no direct way of controlling this if spin $1 / 2$ lattice quarks are used. For this reason we used scalar quarks. The spin spin interaction is well approximated by the two - body Hamiltonian $[43,44]$

$$
\Delta H=-\frac{\pi \alpha_{s}}{6 m_{1} m_{2}} \delta_{3}(\vec{r}) \lambda_{1} \cdot \lambda_{2} \sigma_{1} \cdot \sigma_{2}
$$

where the subscripts 1 and 2 indicate the two different quarks, $\alpha_{\mathrm{s}}$ is the strong coupling constant, $m_{1}$ and $m_{2}$ are the constituent masses of the quarks, the $\lambda^{\prime} \mathrm{s}$ are the appropriate Gell - Mann SU(3) matrices and the $\sigma$ 's are the Pauli spin matrices. Eq.(2) was first successfully used in phenomenological applications by De Rujula, Georgi and Glashow [44]. In first order perturbation theory, $|\Psi(0)|^{2}$ arises because of the delta function in Eq.(2). Our procedure for avoiding the short-distance distortion problem was to measure $\Psi(\mathrm{x})$ at large values of $\mathrm{x}$ and extrapolate the results to $\mathrm{x}=0$. Methods of obtaining quark wavefunctions have been introduced by Velikson, Weingarten and Gottlieb [45]. We have developed an alternative method which is described in ref. $[29,40]$. The fact that wavefunctions scaled indicated that they are physical quantities. Further support for the scalar quark approach has come from the successful computations of the pion and proton electromagnetic form factors $[39,40]$, the lepton width of the $\mathrm{J} / \Psi, \phi$ and $\rho[46]$, and the charge radii of the $\pi^{-}$, the $\mathrm{K}^{-}$and the proton $[39,40]$. 
Individual hadron masses are computed using the equation, Mass $=M_{\text {spin }- \text { ind }}+$ Spin-Spin Correction, where $M_{\text {spin }- \text { ind }}$ is the mass obtained using scalar quarks and the spin-spin correction term is obtained from Eq.(2).

The use of scalar quarks saved a factor of 16 in computer speed and memory needs compared to Wilson fermions. This allowed large lattices $\left(16^{4}\right)$ with long runs and high statistics. The big demands on computer resources made this of non - trivial importance.

These techniques have been applied to accurately compute the masses of mesons and baryons $[41,42]$. The results will be briefly reviewed at the end of this paper (see Tables 3, 4 and 5). Lattice QCD is gradually becoming a powerful computational tool, in which precise numerical results are obtainable.

A theory such as lattice QCD should not only be able to reproduce experimentally known numbers, but go beyond this and predict quantities not yet measured in the laboratory. The purpose of this letter is to make such predictions. We have introduced $\mathrm{a}$ b quark and obtained the masses of baryons and mesons involving beauty. These are the first mass predictions coming from lattice computer simulations.

We used the standard Monte Carlo procedure: We began with a background gauge configuration and thermalized it the equivalent of approximately 1000 sweeps. This was done for each of 19 lattices. These configurations represent typical important contributions to the path integral of the $\mathrm{SU}(3)$ color gauge theory. When quarks propagate throughout these background gluonic mediums, they experience the relativistic forces of QCD. When far apart, they feel a linear potential; when close together they feel a Coulomb-like potential $[32,33]$.

For each of these 19 configurations, we measured quarks propagators for various values of the bare quark masses. In a previous project [41], the physical values of the $\mathrm{u}, \mathrm{s}$ and $\mathrm{c}$ quark masses had been determined. To obtain hadrons with beauty, it was necessary to fix, in addition, the $b$ quark mass. This was done by requiring the mass of the B meson to be its experimental value of $5272 \mathrm{MeV}$. Several runs had to be performed before the precise value of the $b$ quark mass was found.

Having the propagators of the $u, d, s$ and $b$ quarks, it was straightforward to compute the masses of quark - antiquark and tri-quark states. Spin -independent masses $\left(\mathrm{M}_{\text {spin -ind }}\right)$ and $|\Psi(0)|^{2}$ were measured and individual masses were obtained by computing the first order spin correction (Eq.(2)). Table 1 displays the results. Since none of these particles have been observed (except for the B), it is perhaps useful to compare results with quark models $[47,48]$. A typical prediction for the $B^{*}-B$ mass difference is $\left(M_{D}{ }^{*}-M_{D}\right) m_{c} / m_{b}$ or about $50 \mathrm{MeV}$ [47]. We 
obtained $75 \mathrm{MeV}$ (essentially because $|\Psi(0)|^{2}$ has increased significantly in going from the cu system to the bu system). Generally speaking, the substitution of an $\mathbf{S}$ quark for a u quark results in a state whose mass is about $120 \mathrm{MeV}$ heavier. This seems to be the case here: The $F_{b}$ and $F_{b}{ }^{*}$ were about $125 \mathrm{MeV}$ heavier than the $\mathrm{B}$ and $\mathrm{B}^{*}$. From the additive quark model, one might expect the $\Sigma_{\mathrm{b}}$ and $\Lambda_{\mathrm{b}}$ to be about $400 \mathrm{MeV}$ heavier than the B meson, that is $5700 \mathrm{MeV}$. We found a significantly larger value. In the particle data tables [49], weak evidence for the $\Lambda_{b}$ at $5500 \mathrm{MeV}$ has been reported. According to our calculations, it is much too light to be the $\Lambda_{\mathrm{b}}$. The state should weigh $5909 \pm 35 \mathrm{MeV}$. The error bars in Table 1 come from statistical errors in the spin-averaged mass and $|\Psi(0)|^{2}$. For completeness Table 2 provides the parameters which enter the spin -spin term in Eq.(2).

A way of judging the reliability of these predictions is to see how the method has faired with experimentally known data. Table 3 presents the meson masses of ref. [41] which used the same techniques as the present paper. Except for the $\pi$, which is hard to treat with our methods, the results are in good agreement with experiment. Masses above $1 \mathrm{GeV}$ are accurate to $20 \mathrm{MeV}$. Table 4 displays the baryon masses [42]. Masses just above the $1 \mathrm{GeV}$ level agree with experiment to 50 $\mathrm{MeV}$ and the heavier baryons agree to $30 \mathrm{MeV}$. Finally, the reliability of perturbatively treating spin - spin interactions can be tested. Table 5 compares our calculated spin-spin splittings versus experiment. The numbers agree quite well.

This paper has obtained masses predictions, the first of its kind from lattice gauge theories. We feel the present work is a big step forward in making lattice methods a calculational and predictive tool. Because of their accuracy, these mass predictions are useful not only within the context of lattice theories but to experimentalists. In the future, these states will be produced in accelerators. Our results will tell experimentalist where to look.

\section{ACKNOWLEDGEMENTS}

We would like to thank the following people for assitance, support and advice: $O$. Martin, G. Martinelli, F. Rapuano, R. Petronzio, B. Loftus, D. Morrow, R. Giles and $R$. Brower. We are most grateful to Lloyd M. Throndyke, Bobby Robertson, Kent Steiner and John E. Zelenka for access to the ETA Systems Inc 4 Megaword 2 vector pipeline CDC CYBER 205 and to the Colorado State University 2 Megaword vector pipeline CDC CYBER 205. We are grateful to Robert M. Price for continuing support and interest. This research is supported in part by the Control Data Corporation PACER Fellowship (Grant No. 85PCR06) and the Natural Science and Engineering Research Council of Canada (Grant No. NSERC A8420). 
TABLE I

Particle $\begin{gathered}\text { Quark } \\ \text { Content Mass (in MeV) }\end{gathered}$

$\begin{array}{cll}B & u \bar{b} & 5272 \text { (fixed to experiment) } \\ B^{*} & u \bar{b} & 5347 \pm 15 \\ F_{b} & s \bar{b} & 5391 \pm 14 \\ F_{b}^{*} & s \bar{b} & 5471 \pm 17 \\ \Sigma_{b} & u u b & 5973 \pm 20 \\ \Sigma_{b}^{*}\left(3 \frac{2}{2}\right) & u u b & 5990 \pm 25 \\ \Lambda_{b} & u d b & 5894 \pm 35 \\ \Omega_{b}\left(\frac{1,2}{2}\right) & s s b & 6292 \pm 30 \\ \Omega_{b}^{*}(3 / 2) & \text { ssb } & 6305 \pm 30\end{array}$

Predictions for the Masses of Beautiful Hadrons. 
Porticle $\quad B^{*} \quad F_{b} \quad F_{b}^{*} \quad \Sigma_{b}(1 / 2) \quad \Sigma_{b}^{*}(3 / 2) \quad \Lambda_{b} \quad \Omega_{b}(1 / 2) \Omega_{b}^{*}(3 / 2)$

$\begin{array}{ccccccccc}\begin{array}{c}\text { Spin-Averaged } \\ \text { Mass }\end{array} & 5328 & 5451 & 5451 & 5962 & 5962 & 5962 & 6293 & 6293 \\ \begin{array}{c}\text { Constituent } \\ \text { Mass }\end{array} & 4725 & 4725 & 4725 & 4725 & 4725 & 4725 & 4725 & 4725 \\ \begin{array}{c}\text { Constituent } \\ \text { Mass }\end{array} & 489 & 602 & 602 & 620 & 620 & 620 & 785 & 785 \\ \alpha_{s} & .78 & .65 & .65 & .62 & .62 & .62 & .51 & .51 \\ |\Psi(1=2)|^{2} & 19.7 & 31.2 & 31.2 & 10.04 & 10.04 & 10.04 & 6.82 & 6.82 \\ |\Psi(2=3)|^{2} & - & - & - & 9.00 & 9.00 & 9.00 & 11.16 & 11.16 \\ \Delta E_{\text {spin-spin }} & 19 & -60 & 20 & 12 & 28 & -68 & -1 & 12\end{array}$

The Parameters Which Enter in the Spin-Spin Splitting Term of Eq.(2). 
TABLE 3

$\begin{array}{lcc}\text { Meson } & \text { Mass (in MeV) } & \text { Experiment } \\ \pi & 19 \pm 150 & 137 \\ \rho & 809 \pm 60 & 769 \\ K & 476 \pm 90 & 496 \\ K^{*} & 905 \pm 25 & 892 \\ \eta_{s} & 791 \pm 25 & - \\ \varphi^{*} & 1016 \pm 10 & 1020 \\ D & 1854 \pm 20 & 1867 \\ D^{*} & 2000 \pm 15 & 2009 \\ F & 1989 \pm 20 & 1971 \\ F^{*} & 2119 \pm 15 & 2140 \\ \eta_{c} & 2996 \pm 20 & 2981 \\ J_{\Psi} & 3096 \pm 20 & 3097\end{array}$

The Meson Results of Reference [41]. 
TABLE 4

Particle $\begin{gathered}\text { Quark } \\ \text { Content Mass (in MeV) Experiment }\end{gathered}$

$\begin{array}{cccc}\text { Proton } & \text { uud } & 1069 \pm 45 & 939 \\ \Delta(1232) & \text { uud } & 1262 \pm 45 & 1232 \\ \Sigma & \text { uus } & 1249 \pm 40 & 1193 \\ \Sigma^{*}(1385) & \text { uus } & 1441 \pm 50 & 1385 \\ \Lambda & \text { uds } & 1164 \pm 60 & 1116 \\ \equiv & \text { uss } & 1338 \pm 55 & 1318 \\ \Xi^{*}(1530) & \text { uss } & 1575 \pm 45 & 1535 \\ \Omega & \text { sss } & 1694 \pm 35 & 1672 \\ \Sigma_{c} & \text { uuc } & 2431 \pm 20 & 2450 \\ \Sigma_{c}^{*}(3 / 2) & \text { uuc } & 2594 \pm 45 & \text { not yet observed } \\ \Lambda_{c} & \text { udc } & 2282 \pm 60 & 2282 \\ \Omega_{c}\left(1 \frac{2}{2}\right) & \text { ssc } & 2727 \pm 25 & \text { not yet observed } \\ \Omega_{c}^{*}\left(3 \frac{2}{2}\right) & \text { ssc } & 2768 \pm 35 & \text { not yet observed }\end{array}$

The Baryon Mass Results of Reference [42]. 
TABLE 5

\begin{tabular}{|c|c|c|}
\hline Particles & $\begin{array}{c}\text { Calculated } \\
\text { Splitting } \\
\text { (MeV) }\end{array}$ & $\begin{array}{c}\text { Experimental } \\
\text { Splitting } \\
\text { (MeV) }\end{array}$ \\
\hline$p-\pi$ & $790 \pm 150$ & 632 \\
\hline$K^{*}-K$ & $429 \pm 80$ & 396 \\
\hline$D^{*}-D$ & $146 \pm 30$ & 142 \\
\hline$F^{*}-F$ & $130 \pm 25$ & 169 \\
\hline$J / \Psi-\eta_{c}$ & $100 \pm 20$ & 116 \\
\hline$\Delta-$ proton & $193 \pm 35$ & 293 \\
\hline$\Sigma^{*}(1385)-\Sigma$ & $192 \pm 35$ & 192 \\
\hline$\Sigma-\Lambda$ & $86 \pm 15$ & 85 \\
\hline$\Xi^{*}(1530)-\equiv$ & $237 \pm 45$ & 215 \\
\hline$\Sigma_{c}^{*}(3 / 2)-\Sigma_{c}$ & $73 \pm 13$ & not yet observed \\
\hline$\Sigma_{c}-\Lambda_{c}$ & $148 \pm 25$ & 168 \\
\hline$\left(3 / 2^{+}\right)-\Omega_{c}(1 / 2)$ & $41 \pm 8$ & not yet obser \\
\hline
\end{tabular}

Comparison of Computed Spin -Spin Splittings Versus Experiment. 


\section{REFERENCES}

[1] K. G. Wilson, Phys. Rev. D10 (1974) 2445.

[2] M. Creutz, Phys Rev. D21 (1980) 2308;

Phys. Rev. Lett. 45 (1980) 313.

[3] M. Creutz, L. Jacobs and C. Rebbi, Phys. Rev. D20 (1979) 1915.

[4] H. Hamber and G. Parisi, Phys. Rev. Lett. 47 (1981) 1797.

[5] D. H. Weingarten, Phys. Lett. 109B, (1982) 57.

[6] E. Marinari, G. Parisi and C. Rebbi, Phys. Rev. Lett. 47 (1981) 1795.

[7] A. Hasenfratz, P. Hasenfratz, Z. Kunszt, and C. B. Lang, Phys. Lett. 110B (1982) 289.

[8] F. Fucito G. Martinelli, C. Omero, G. Parisi, R. Petronzio, and F. Rapuano, Nucl. Phys. B210 [FS6] (1982) 407.

[9] C. Bernard, T. Draper, K. Olynyk and M. Rushton, Nucl. Phys. B220 [FS8] (1983) 508.

[10] R. Gupta and A. Patel, Phys. Lett. 124B (1983) 94;

Nucl. Phys. B226 (1983) 152.

[11] D. H. Weingarten, Nucl. Phys. B215 [FS7] (1983) 1.

[12] H. Hamber and G. Parisi, Phys. Rev. D27 (1983) 208.

[13] H. Lipps, G. Martinelli, R. Petronzio and F. Rapuano, Phys. Lett. 126B (1983) 250.

[14] J. P. Gilchrist, G. Schierholz, H. Schneider and M. Teper, Nucl. Phys. B248 (1984) 29.

[15] A. Billoire, E. Marinari and R. Petronzio, Nucl. Phys. B251 [FS13] (1985) 141.

[16] A. Billoire, R. Lacaze, E. Marinari and A. Morel, SACLAY preprint SPhT 84/92 (Sept. 1984).

[17] D. Barkai, K. J. M. Moriarty and C. Rebbi, Phys. Lett. 156B (1985) 385. 
[18] A. Konig, K. H. Mutter and K. Schilling, Phys. Lett. 147B (1985) 145;

Phys. Lett. 157B (1985) 421.

[19] C. Bernard, T. Draper, K. Olynyk, and M. Rushton, Phys. Rev. Lett. 49 (1982) 1076.

[20] G. Martinelli, G. Parisi, R. Petronzio and F. Rapuano, Phys. Let. 116B (1982) 434.

[21] R. C. Brower, M. B. Gavela, R. Gupta and G. Maturana, Phys. Rev. Lett. 53 (1984) 1318.

[22] N. Cabibbo, G. Martinelli and R. Petronzio, Nucl. Phys. B244 (1984) 381.

[23] S. Gottlieb, P. B. Mackenzie, H. B. Thacker and D. Weingarten, Fermilab preprint pub-84/98-T (May, 1985).

[24] K. C. Bowler, G. S. Pawley, D. J. Wallace, E. Marinari and F. Rapuano, Nucl. Phys. B220 [FS8] (1983) 137.

[25] H. Hamber, Phys. Rev. D31 (1985) 586.

S. Samuel and O. Martin, Phys. Rev. D31 (1985) 1768.

[26] K. G. Wilson in "Recent Developments in Gauge Theories", (Cargese, 1979), (Plenum Press, New York, 1980).

[27] L. Susskind, Phys. Rev. D16 (1977) 3031.

[28] H. Nielsen and M. Minomiya, Nucl. Phys. B185 (1981) 20.

[29] O. Martin, K. Moriarty and S. Samuel, Nucl. Phys. B261 (1985) 433.

[30] G. Bhanot and M. Creutz, Phys. Rev. D24 (1981) 3213;

G. Bhanot, Phys. Lett. 108B (1982) 337.

[31] O. Martin, K. Moriarty and S. Samuel, Phys. Lett. 153B (1985) 87.

[32] S. Otto and J. D. Stack, Phys. Rev. Lett. 52 (1984) 2328.

[33] D. Bakai, K. J. Moriarty and C. Rebbi, Phys. Rev. D30 (1984) 1293.

[34] F. Gutbrod, P. Hasenfratz, Z. Kunszt and I. Montvay, Phys. Lett. 128B (1983) 415.

[35] F. Karsch and P. Petronzio, Phys. Lett. 139B (1984) 403. 
[36] A. D. Kennedy, J. Kuti, S. Meyer and B. J. Pendleton, Phys. Rev. Lett. 54 (1985) 87.

[37] K. C. Bowler, D. L. Chambers, A. Kenway, R. D. Kenway, G. S. Pawley and D. J. Wallace, Nucl. Phys. B240 (1984) 213.

[38] K. Moriarty and S. Samuel, "On the Constituent Masses of Quarks", (CCNY preprint, March, 1985).

[39] K. Moriarty and S. Samuel, "A Lattice QCD Calculation of Mesonic Electromagnetic Form Factors", (CCNY preprint, April, 1985).

[40] S. Samuel and K. Moriarty, "Electromagnetic Form Factors of Hadrons from Scalar Lattice QCD", work in progress.

[41] S. Samuel and K. Moriarty, Phys. Lett. 158B (1985) 437.

[42] S. Samuel and K. Moriarty, "Precise Baryon Mass Calculations from Scalar Lattice QCD", (CERN - TH. -4271/85 preprint, October, 1985).

[43] E. Fermi, Mem. Accad. d'Italia I, (Fis) (1930) 139.

[44] A. De Rugula, H. Georgi and S. L. Glashow, Phys. Rev. D12 (1975) 142.

[45] B. Velikson and D. Weingarten, Nucl. Phys. B249 (1985) 433;

S. Gottlieb, "Portrait of a Proton", (UCSD preprint 10P10-248, July, 1985).

[46] S. Samuel, "A Lattice QCD Calculation of Vector Meson Decay into Lepton Pairs", (CCNY preprint, January, 1985).

[47] C. Quigg, "Models for Hadrons", Les Houches, 1981 (North Holland, Amsterdam, 1983);

[48] D. Flamm and F. Schoberl, "Introduction to the Quark Model of Elementary Particles", (Gordon and Breach, New York, 1982).

[49] Particle Data Group, Review of Particle Properties, Rev. Mod. Phys. 56 (1984). 